\title{
July 2018 Pulmonary Case of the Month
}

Anjuli M. Brighton, $M B, B C h, B A O$

Mayo Clinic Arizona

Scottsdale, AZ USA

\section{History of Present IIIness}

An 81 -year-old gentleman was admitted for syncope. He had felt unwell for one month. His recent illness started with the "flu". He had lingering productive cough, low volume hemoptysis and felt very fatigued. After a coughing episode he apparently lost consciousness and was taken to the emergency department.

\section{Past Medical History, Social History and Family History}

$\mathrm{He}$ has a past medical history of hypertension, glaucoma, diverticulosis and COPD. He was taking only antihypertensives including a diuretic. He has a 30 pack-year history of smoking but quit 10 years ago.

\section{Physical Examination}

- Normotensive

- Tachypneic

- SpO2 $96 \%$ on $2 \mathrm{~L}$ NC

- Afebrile

- Diffuse wheezing, diminished at $L$ base

- Irregularly irregular heart rate

Which of the following are indicated at this time?

1. Chest x-ray

2. Complete blood count (CBC)

3. Electrocardiogram (EKG)

4. 1 and 3

5. All of the above 


\section{Correct \\ 5. All of the above}

His CBC showed a borderline eosinophilia at 6\% (normal range 0-6\%) but was otherwise unremarkable. Electrolytes and liver function tests were also ordered which were normal. EKG confirmed the presence of atrial fibrillation. Chest X-ray is below (Figure 1).

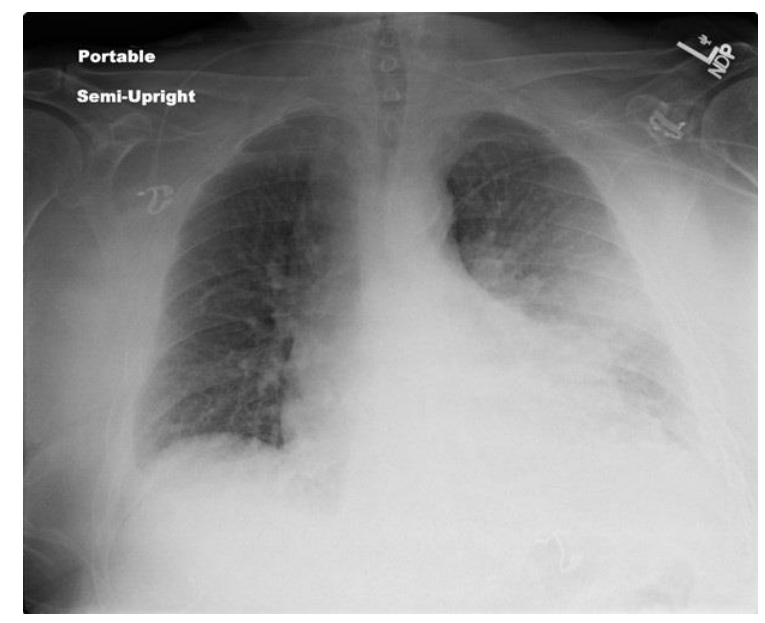

Figure 1. Portable PA of the chest performed on admission.

What should be done at this time?

1. Brain naturetic peptide (BNP) or NT-pro B-type natriuretic peptide (NT pro BNP)

2. CT scan of the chest

3. Echocardiogram

4. 1 and 3

5. All of the above 


\section{Correct! \\ 5. All of the above}

The chest x-ray shows cardiomegaly, increased density in the left lower lung and probable left pleural effusion. Given the increased size of the heart and the possible pleural effusion and apparent recent development of atrial fibrillation congestive heart failure is a possibility. BNP and NT-proBNP are markers of atrial and ventricular distension due to increased intracardiac pressure. Our patient's NT-proBNP was markedly elevated at $30,000 \mathrm{pg} / \mathrm{ml}$ (normal $10-138$ at age $>83$ years).

Echocardiography showed a left ventricular ejection fraction of $38 \%$ with right ventricular motion abnormality and right ventricular dysfunction.

To better define the abnormalities on chest $\mathrm{x}$-ray, a CT scan of the thorax was performed (Figure 2).

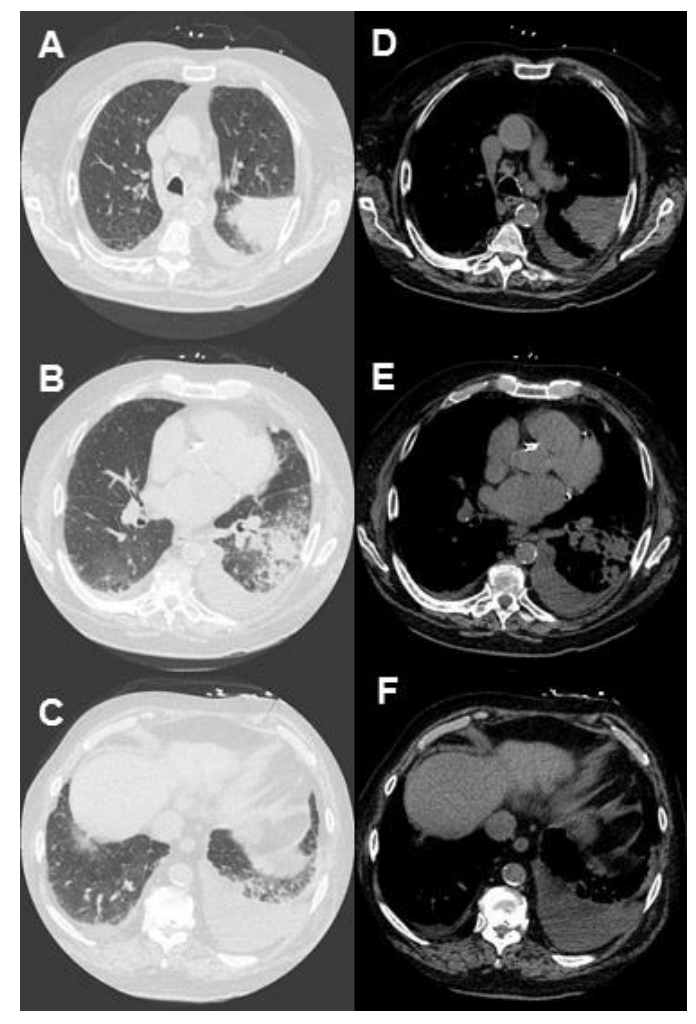

Figure 2. Representative images from the thoracic $C T$ in lung windows $(A-C)$ and soft tissue windows (D-F).

Which of the following should be done next?

1. Coccidioidomycosis antigen

2. Diuresis for congestive heart failure

3. Thoracentesis

4. 1 and 3

5. All of the above 


\section{Correct!}

\section{1 and 3}

Here in Arizona coccidioidomycosis (Valley Fever) seems to be in nearly every thoracic differential. A left-sided consolidation and pleural effusion in a patient who has a chronic or subacute process would be compatible with coccidioidomycosis, especially given the eosinophilia. In 2005 Crum and Wallace (1) reviewed 162 cases of coccidioidomycosis and found $28 \%$ had peripheral blood eosinophilia (mean 6\%, range 0-44\%). In 2013 Alzoubaidi et al. (2) found similar results also in 162 patients with an average of $6 \%$ eosinophils in patients with coccidioidomycosis. However, in their series $71 \%$ had peripheral blood eosinophilia and $53 \%$ had having bronchoalveolar lavage eosinophilia.

The elevated NT-proBNP suggests congestive heart failure. Although it is often stated that isolated left-sided pleural effusions are unusual in heart failure, this concept was challenged by Woodring (3). He found that a left-sided pleural effusion is not an atypical finding in congestive heart failure. However, heart failure would not explain the hemoptysis or left-sided focal consolidation. Therefore, it was felt that diuresis was premature until thoracentesis was performed.

Thoracentesis removed $800 \mathrm{~mL}$ of yellow fluid. Major findings were as below:

- $\mathrm{pH} 7.49$

- Total protein $2.2 \mathrm{~g} / \mathrm{dL}$ (serum 6.7)

- LDH $119 \mathrm{U} / \mathrm{L}$ (serum 236, normal range 111-222)

- Cell count 700 cells $/ \mathrm{mL}$

- Differential

- $22 \%$ neutrophils

- $0 \%$ eosinophils

- $52 \%$ lymphocytes

- $18 \%$ macrophages

- Coccidioidomycosis by EIA and PCR negative

- Cytology negative for malignancy

Which of the following should be done next?

1. Diuresis. No further work up is required.

2. Lung PET scan

3. Pleuroscopy

4. 1 and 3

5. All of the above 


\section{Correct! \\ 2. Lung PET scan}

The effusion is transudative by Light's criteria (pleural fluid/serum protein $<0.5$ and pleural fluid/serum LDH $<0.6$ or $<2 / 3$ the upper limit of the serum normal, 4$)$. It is unusual for patients with lung cancer to present with a transudative effusion and when it occurs it is most often seen with coexisting congestive heart failure (5). We were still troubled that the clinical history was not totally explained by congestive heart failure, the patient's hemoptysis and the focal lung consolidation or mass. A pleuroscopy is reasonable but probably overly invasive at this juncture. Therefore, a lung PET scan was performed which showed increased tracer uptake in the left lung (Figure 3).

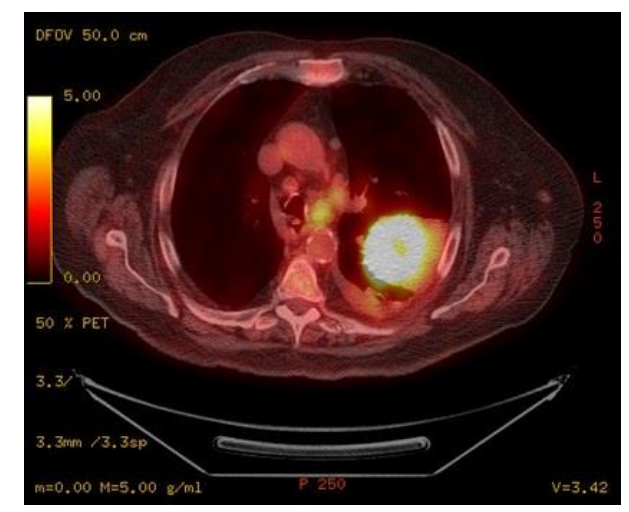

Figure 3. Representative image from lung PET scan showing increased uptake of FDG (fluorine-18 combined with deoxy-glucose) in the left lung.

What should be done next?

1. Bronchoscopy

2. Continue diuresis. Increased FDG is seen in congestive heart failure.

3. Continue diuresis. The increased FDG uptake is explained by the patient's high glucose.

4. 1 and 3

5. All of the above 


\section{Correct! \\ 1. Bronchoscopy}

The increased uptake of FDG is not a normal finding in heart failure. High glucose levels can decrease the uptake of FDG in a lung PET scan, not increase it as seen in this patient. Therefore, bronchoscopy is the only reasonable choice. The results demonstrated a tumor in the left lower lobe (Figure 4).

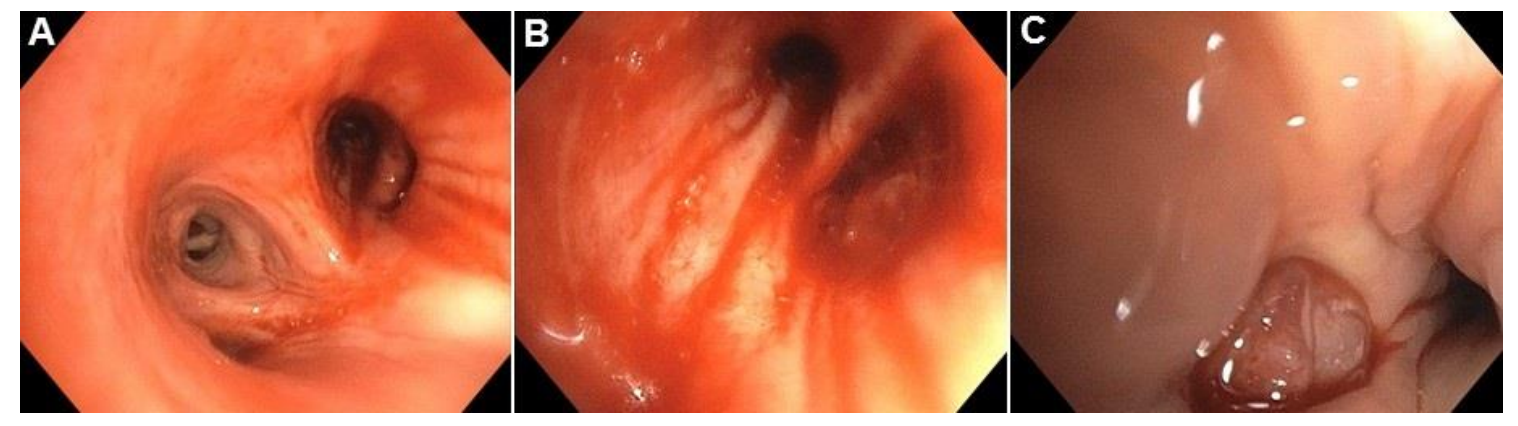

Figure 4. Bronchoscopic views in the (A) left lower lobe bronchus, (B) left lobe subsegmental bronchi and $(C)$ subsegmental bronchus showing the presence of a tumor.

Biopsy of the lesion showed poorly differentiated squamous cell cancer which was PDL1 negative. The patient was offered chemotherapy and radiation therapy but opted for radiation therapy alone due to his debilitated condition.

\section{References}

1. Crum NF, Wallace MR. Laboratory values are predictive of disseminated coccidioidomycosis. Infect Dis Clin Pract (Baltim Md). 2005;13(2):68-72. [CrossRef]

2. Alzoubaidi MSS, Knox KS, Wolk DM, Nesbit LA, Jahan K, Luraschi-Monjagatta C. Eosinophilia in coccidioidomycosis. Am J Resp Crit Care. 2013;187:A5573. [Abstract]

3. Woodring $\mathrm{JH}$. Distribution of pleural effusion in congestive heart failure: what is atypical? South Med J. 2005 May;98(5):518-23. [CrossRef] [PubMed]

4. Light RW, Macgregor MI, Luchsinger PC, Ball WC Jr. Pleural effusions: the diagnostic separation of transudates and exudates. Ann Intern Med. 1972 Oct. 77(4):507-13. [CrossRef] [PubMed]

5. Ashchi M, Golish J, Eng P, O'Donovan P. Transudative malignant pleural effusions: prevalence and mechanisms. South Med J. 1998 Jan;91(1):23-6. [CrossRef] [PubMed] 